\title{
New Zealand Hybrid Rabbit
}

National Cancer Institute

\section{Source}

National Cancer Institute. New Zealand Hybrid Rabbit. NCI Thesaurus. Code C77110.

A rabbit that is derived from a cross between two different New Zealand strains of determinate or indeterminate coat genotype. The New Zealand hybrid is commonly utilized in biomedical research, in a variety of studies including developmental toxicity studies, toxicology studies, asthma research, and antibody production. 